\title{
A Spin Glass Based Framework to Untangle Fiber Crossing in MR Diffusion Based Tracking
}

\author{
Y. Cointepas ${ }^{1}$, C. Poupon ${ }^{2}$, D. Le Bihan ${ }^{1}$, and J.-F. Mangin ${ }^{1}$ \\ 1 UNAF, Service Hospitalier Frédéric Joliot, CEA, France \\ cointepa@shfj.cea.frhttp://www-dsv.cea.fr/ \\ ${ }^{2}$ General Electric Medical Systems, Clinical Software Applications, Buc, France \\ cyril.poupon@med.ge.com
}

\begin{abstract}
We propose a general approach to the reconstruction of brain white matter geometry from diffusion-weighted data. This approach is based on an inverse problem framework. The optimal geometry corresponds to the lowest energy configuration of a spin glass. These spins represent pieces of fascicles that orient themselves according to diffusion data and interact in order to create low curvature fascicles. Simulated diffusion-weighted datasets corresponding to the crossing of two fascicle bundles are used to validate the method.
\end{abstract}

\section{Introduction}

A number of algorithmic approaches have been recently proposed to study anatomical connectivity from MR diffusion-weighted data. For most of these approaches, the putative fascicles are revealed by the step by step reconstruction of highest diffusion $3 \mathrm{D}$ trajectories [11,2]. The reconstruction is performed from a vector field made up of the local directions of highest diffusion, usually the diffusion tensor first eigenvector. Unfortunately, this approach is not robust to spurious local directions, which induce erroneous forks in the tracking process. Since partial volume averaging at the level of fiber crossing is bound to generate such spurious highest diffusion directions $[12,3]$, more robust tracking schemes have to be designed. A direction of research consists of simulating large scale diffusion processes throughout white matter, either at the random walk level [5], or at the macroscopic level using partial differential equation frameworks [8]. Such methods provide for each given input area a map of connectivity probability related for instance to the time required for some information to reach any brain area. Tracking information propagation streamlines may allow the mapping of the putative bundle trajectories stemming from this input area including possible forks. One new diffusion process, however, has to be simulated for each new input area. A second class of approaches relies on regularization principles leading to search for the optimal path between a pair of input points [10], or for the optimal fascicle map linking a set of points [9], combining diffusion data and a priori knowledge on fascicle curvature.

In this paper, we extend the global approach mentioned above [9], casting it into a global inverse problem framework. This framework leads to infer the fascicle map with highest likelihood from a set of observations about the water diffusion process occuring into brain white matter. Such a fascicle map is made up of a set of inter-connected fascicle pieces. The whole fascicles can be automatically reconstructed from this map 
as paths following the links defined between these fascicle pieces. The fascicle map includes branching locations, which results in more tracking efficiency than using a path by path approach, because the chains of fascicle pieces included in several paths are investigated only one time during optimization. Once the optimal fascicle map has been inferred, an exhaustive list of pairs of points of the white matter surface linked by a putative fascicle can be provided at low cost.

The optimality of the fascicle map is defined as a trade-off between local information on voxel micro-structure provided by diffusion data and a priori information on the low curvature of plausible fascicles. This trade-off is obtained from the minimization of a global measure of the likelihood of a fascicle map knowing the diffusion data set. This likelihood is made up of two kind of terms. The first ones assess the quality of the fit between the local diffusion data and the related local pieces of fascicle. The second ones assess the contextual plausibility of the local configurations of fascicle pieces. Contextual plausibility is used to detect and regularize the spurious fascicle local directions induced by corrupted or ambiguous diffusion data. This measure is modeled using the ideas that most of the fascicles have a rather low bending in anatomical dissections and that a fascicle cannot end up inside white matter.

Our framework stems from the field of spin glasses developped in statistical physics. During our previous works, the fascicle map was including only one fascicle piece per voxel, which was unsufficient to deal correctly with bundle crossing. Moreover, only diffusion tensor models had been considered, while MR physics research should lead to much more informative high angular resolution models $[3,10]$. In this paper, we show that the spin glass based approach can be extended to deal with more or less accurate diffusion data and can embed the possible existence of some crossings inside voxels. The extended model behaviour is explored using simulated data in order to get rid of the MR physics problems that have still to be overcome to understand actual MR diffusion data [1].

\section{Framework}

Inverse problem: The reconstruction of the white matter fascicle geometry from diffusionweighted data may be considered as a standard inverse problem. Like many other inverse problems, fascicle map inference has to deal with acquisition artifacts and coarse data sampling. The choice of the trade-off between signal to noise, spatial and angular resolutions is important relatively to the accuracy of the fascicle map that can be reconstructed. If the fascicle map resolution is too high relatively to the relevant information included in the data, the inverse problem becomes ill-posed, which means that two different acquisitions of the same brain will yield two very different fascicle maps simply because of noise and partial volume. Any single wrong local diffusion data interpretation, indeed, may create a spurious fascicle in the map while splitting an actual one. Voxels including crossing fibers are especially difficult to deal with and may lead to different fascicle reconstructions according to the data spatial and angular sampling. It should be understood also that several different local fiber configurations may provide the same diffusion data. For instance, a flat tensor may correspond to a crossing or to 
a "fan shaped" fascicle. Hence, there is a need for the problem to be formulated in a way that gives the same unique solution with different acquisitions, at least for the same sampling level.

A standard approach to deal with ill-posed problems relies on the regularization principle: a priori knowledge on the regularity of the most plausible solutions allows the distinction between relevant information and noise. The family of methods proposed in the following uses the reasonable hypothesis that in case of ambiguity, the most plausible fascicle trajectory is endowed with the lowest curvature. This hypothesis and the additional knowledge that a fascicle should not end inside white matter allows the method to extrapolate the reliable fascicle segments inferred from the areas of unequivocal diffusion data to more problematic white matter areas.

Fascicle maps and spin glasses: As mentioned above, the choice of the fascicle map resolution has to be consistent with the amount of relevant information embedded in the diffusion data. Hence, the following methods aim at recovering maps including only a limited number of fascicles into each diffusion data voxel. Once the fascicle map domain has been defined, a representation has to be devised for the local pieces of fascicles. Each piece will have some degrees of freedom, which will lead to set out the inverse problem as an optimization driven issue. While a lot of different approaches may be figure out, we focus in the following on an analogy with spin glasses. Therefore, the pieces of fascicles will be called spins. Whatever the nature of the diffusion data set, it will be embedded into our framework as a non stationary external magnetic field acting on the spin orientations. The a priori knowledge on the fascicle map geometry (low fascicle curvature, no dead ends inside white matter) will be embedded as interactions between neighboring spins. The solution to the fascicle map inverse problem will be defined as the minimal energy configuration of the spin glass. The family of methods proposed in this paper includes more or less sophisticated variants related to the kind of spins that live inside the domain voxels. The simplest model, which has been used during our previous experiments [9], puts one compass needle like spin in the center of each voxel. In this paper, we introduce more sophisticated models that either relax the spin localization in order to improve the fascicle trajectory sampling, or put several spins inside each voxel in order to deal with fascicle crossing.

The external magnetic field models: For each voxel $M$ of the fascicle domain, information on local water mobility inferred from the diffusion data can be used to build models which provide for any direction of space the likelihood of the existence of a fascicle. These models can then be converted into a virtual diffusion based local potential $P_{D}^{M}$ acting on the orientations $\boldsymbol{v}_{i}^{M}$ of the spins $i$ located inside $M$. Hence, the virtual potential field made up of these local potentials will act on the simulated spin glass as a non stationary magnetic field. Various likelihood models can be devised according to the nature of the diffusion data. As matter stands when using DTI data, these models are directly related to the hypothesis that the diffusion coefficient is higher in the fascicle direction than in neighboring directions. Recent experiments including higher angular resolution data, however, have proved that this hypothesis was not always true at the level of crossings $[12,3]$. Hence, a better understanding of the crossing and compartment issues might lead to better likelihood models in the future [7,1]. 
When the spin glass is embedded into such an external potential field, the spin orientations rotate in order to reach a minimum of energy, which can be simulated using a minimization algorithm. Without any spin interaction, a minimum for the whole glass is made up of minima in each voxel. With tensor data, the spin align themselves along the tensor eigenvector associated with the largest eigenvalue. Hence, the resulting spin glass configuration is equivalent to the vector field used by standard tracking algorithms [2]. In the following, spin interaction potentials are added into the glass energy in order to introduce the a priori knowledge about the low curvature of most of the fascicles. Hence, spin glass energy minima will correspond to a global trade-off between two different kinds of forces.

The interaction model: At the resolution of standard diffusion data, the geometry of the fascicle map may be related to the geometry of spaghetti plates. Therefore, the interaction potential that will embed a low curvature constraint for the fascicles is inspired by a simple model of the local bending energy accumulated by spaghetti during cooking, namely a quadratic potential based on the spaghetti local curvature [9]. Then, a spaghetti potential $P_{S}^{M}(i)$ is defined to embed the interactions between each spin $i$ located into voxel $M$ and the neighboring spins, namely the set $\mathcal{N}_{i}{ }^{M}$ made up of all the other spins located into $M$ and of the spins located in $M$ neighboring voxels in the fascicle map domain. The neighboring spin set $\mathcal{N}_{i}^{M}$ is split first into forward and backward subsets from the spin $i$ orientation $\boldsymbol{v}_{i}$. Then one neighboring spin is selected in each half-neighborhood (respectively $f(i)$ and $b(i)$ ) in order to create a local fascicle trajectory $b(i)-i-f(i)$ endowed with the lowest possible curvature. Note that whatever the relative orientations of $\boldsymbol{v}_{b(i)}, \boldsymbol{v}_{i}$, and $\boldsymbol{v}_{f(i)}$, a putative fascicle has to be defined because of our assumption that the fascicles can not lead to dead ends inside white matter. Each time that one half-neighborhood is empty, however, no best neighboring spin can be defined and the fascicle is supposed to leave white matter. Therefore, no bending constraint is added into $P_{S}^{M}(i)$ in that direction. Whatever the spin glass model, the lowest energy configurations induced by the spaghetti interaction potentials are made up of straight fascicles. Such configurations, indeed, have a null energy and correspond to spaghetti sets without any cooking.

Global energy and minimization: Diffusion based potentials and bending energy based spin interactions are gathered into a global energy $E$ which is defining the solution to the inverse problem as the lowest energy configuration:

$$
E=\sum_{M} \sum_{i} P_{D}^{M}(i)+\alpha \sum_{M} \sum_{i} P_{S}^{M}(i)
$$

where $\alpha$ is a positive rigidity constant which balances the influence of the a priori knowledge on the fascicle low curvature. The solution is obtained using either deterministic or stochastic minimization, according to the quality of the initialization that can be provided. The fascicle map model is more flexible than the path by path method proposed by Tuch et al. [10], because chains of linked spins may split and merge during the minimization. Split and merge operations create additional paths throughout the energy landscape, which highly reduces the influence of initialisation and local minima during optimization. 


\section{Experiments}

In the following, some experiments are proposed to show the advantage of the glass models including several spins into each voxel to deal with fiber crossing. All these experiments rely on simulated data to discard potential difficulties induced by the current weaknesses of the diffusion models used to interpret high angular resolution data $[10,3,1]$. Our goal is to provide a tracking model that will be ready to untangle fiber crossing when current MR physics problem will have been overcome. The lack of data obtained from some phantoms with known fiber geometry, indeed, prevents a reliable use of actual data to test tracking methods.

\section{Simulation of the diffusion driven local likelihood:}

In order to generate a simulated dataset including in each voxel the likelihood of the presence of a fascicle in any orientation of space, a virtual bundle crossing geometry is created (see Fig. 1). For each voxel of the mask corresponding to this geometry, the proportion of each bundle contributing to the partial volume effect is computed. Then, a direction set supposed to correspond to the direction set of the MR sequence is chosen. In order to simulate the diffusion based MR signal attenuation in one direction $\boldsymbol{d}$, a different weighted sum of tensors [6] is used within each voxel $v$ to represent the contribution of each fiber bundle:

$$
A_{v}(\boldsymbol{d})=\left(1-f_{v}^{1}-f_{v}^{2}\right) \cdot e^{\boldsymbol{d}^{T} \cdot \boldsymbol{D}^{\mathbf{o}} \cdot \boldsymbol{d}}+f_{v}^{1} \cdot e^{\boldsymbol{d}^{T} \cdot \boldsymbol{D}^{1} \cdot \boldsymbol{d}}+f_{v}^{2} \cdot e^{\boldsymbol{d}^{T} \cdot \boldsymbol{D}^{2} \cdot \boldsymbol{d}},
$$

where $f_{v}^{1}$ end $f_{v}^{2}$ denote the volume fraction of each fiber bundle $\left(f_{v}^{1}+f_{v}^{2} \leq 1\right), \boldsymbol{D}^{\mathbf{0}}$ is an isotropic tensor representing pure water, $\boldsymbol{D}^{\mathbf{1}}$ and $\boldsymbol{D}^{\mathbf{2}}$ are highly anisotropic tensors adapted to each fiber bundle direction.

For the following experiments, we have chosen to simulate the standard situation where diffusion data stem from the single tensor model, which is in our opinion unsufficient to untangle fiber crossings in a robust way. Nevertheless, this simulation is sufficient to show the superiority of multi-spin per voxel glasses and is closer to the current understanding about what can be obtained from MR diffusion. The simulated high angular resolution diffusion dataset (40 directions) is used to fit in each voxel $v$ a single tensor, from which is computed the likelihood function proposed in [9]. For a direction $\boldsymbol{d}$ :

$$
\text { likelihood }_{v}(\boldsymbol{d})=\frac{\left(e^{\boldsymbol{d}^{T} \cdot \boldsymbol{D}_{v} \cdot \boldsymbol{d}}-\lambda_{v}^{\max }\right)^{2}}{\left\|\boldsymbol{D}_{v}\right\|},
$$

where $\boldsymbol{D}_{v}$ is the tensor estimated in voxel $v$ and $\lambda_{v}^{\max }$ is the maximum eigen value of $\boldsymbol{D}_{v}$. Figure 1 shows that anisotropic likelihoods are obtained in voxels containing homogenous fiber directions and, because of partial volume effect, flat likelihoods are obtained in voxels corresponding to fascicle crossing.

\section{Fascicle map reconstruction:}

In order to illustrate the problems that can arise in fiber tracking methods that consider only one fiber direction per voxel, we present the reconstructions obtained putting one or two spins in each voxel. These reconstructions stem from the minimisation of the global energy presented in section 2 with a stochastic method based on the Gibb's sampler [4]. Using only one spin per voxel cannot lead to a correct crossing representation. 

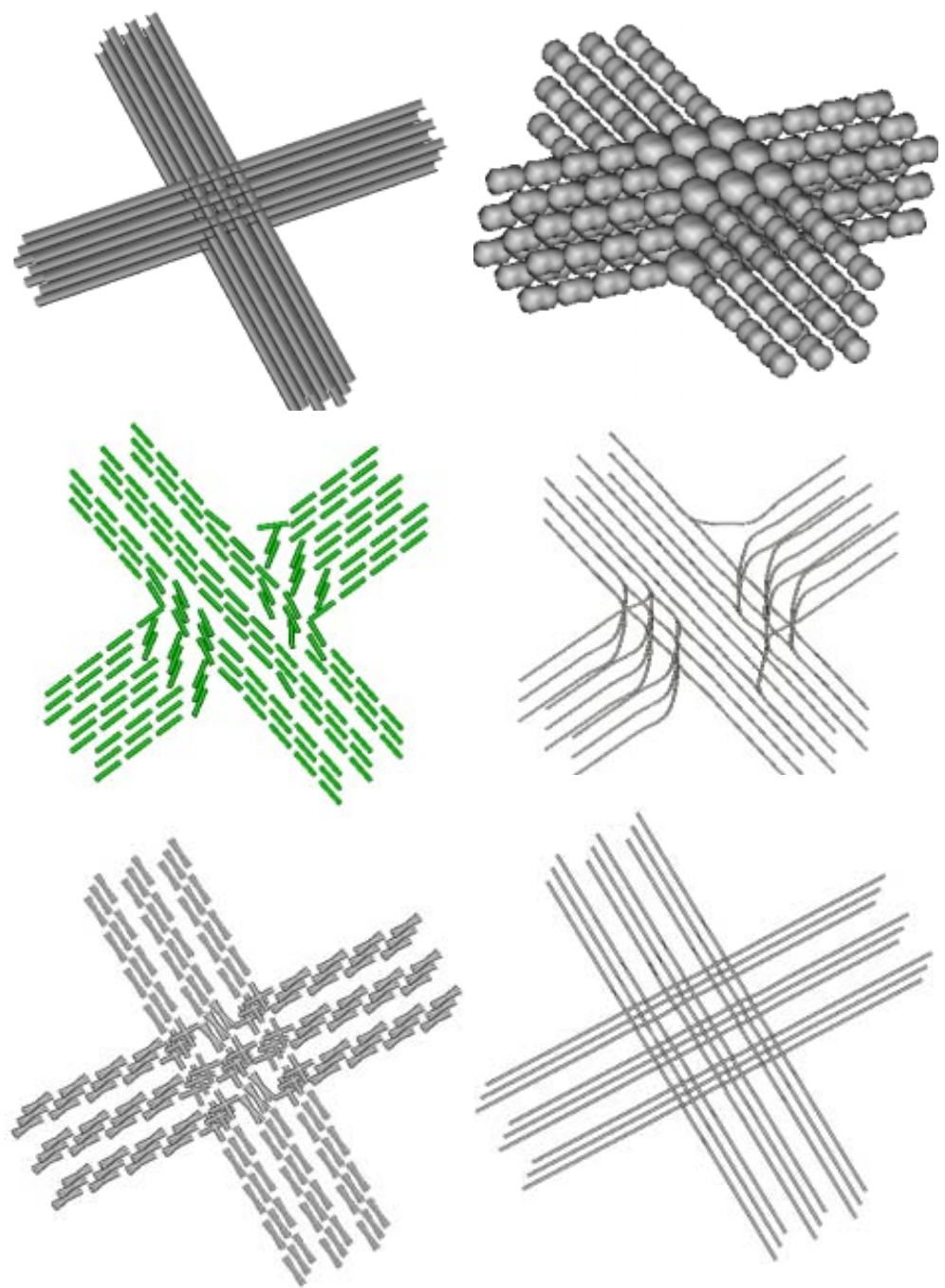

Fig. 1. Top left: a virtual bundle crossing. Top right: the corresponding tensor based orientation likelihood functions. For the sake of understanding, $1-$ likelihood $_{v}(d)$ is actually shown. Middle Optimal glass configuration using one spin per voxel. One of the two bundles wins while the other is broken into two parts, which leads to spurious tracts. Down Optimal glass configuration using two spins per voxel. The crossing is correctly reconstructed.

Therefore, during the minimization process, one bundle wins, whereas the other leads to spurious connections (cf. Fig. 1). In return, with two spins located into each voxel, the simulated geometry is correctly inferred by the minimisation. The spins located into one single bundle area are all oriented in the bundle direction, whereas in the crossing 
the spins are oriented along the two directions. Hence, the resulting tracts correspond to the initial geometry (cf. Fig. 1).

While the previous experiment has shown the advantage of more complex spin glasses, untangling a complex fascicle geometry will require diffusion data with higher angular resolution, which is beyond the scope of the paper. Higher angular resolution, however, will be reached at the cost of noisier data. Hence, a complex issue will be related to the choice of the optimal trade-off between this angular resolution and the number of degrees of freedom used to interpret and smooth them. With the 6 degrees of freedom single tensor model, for instance, noisy data give smooth likelihood functions. A lot of information, unfortunately, is lost about the fiber orientation into crossing areas.

Dealing with higher angular resolution models, however, requires the resolution of a local inverse problem at the level of each voxel. Indeed, converting a high angular resolution diffusion-weighted dataset into a likelihood potential is far to be straightforward. First, a model of the diffusion process has to be chosen, for instance the multicompartment model underlying equation 3. Second the parameters of this model have to be estimated from noisy data (fractions and tensors), which is a difficult non linear estimation problem. Finally, a likelihood model has to be inferred from this MR physics model, which has to be related to the estimation variance. Without a good understanding of this process, which has to be adressed in our opinion by any tracking method, experimenting the spin glass framework with higher angular resolution models may be meaningless, which explains our current choice. This inverse problem framework, however, is sufficiently versatile to be adapted to such new physical models in a very intuitive way.

\section{Conclusion}

In this paper, we have formalized the reconstruction of a putative fascicle map from diffusion-weighted data as a global inverse problem. The proposed framework is flexible enough to be adapted to foreseeable evolutions of MR acquisition schemes. In our opinion, the inverse problem point of view will help to clarify what reliable information can be extracted from a diffusion-weighted dataset. More and more informative connectivity matrices will be computed from the reconstructed fascicle maps, leading to a very attractive new field of research for neurosciences, ranging from pure graph theory to brain growth or new insights into connectivity related pathologies.

\section{References}

1. P. J. Basser. Relationships between diffusion tensor and q-space MRI. MRM, 47(2):392-7, 2002.

2. P. J. Basser, S. Pajevic, C. Pierpaoli, J. Duda, and A. Aldroubi. In vivo fiber tractography using DT-MRI data. MRM, 44(4):625-632, 2000.

3. L. R. Frank. Anisotropy in high angular resolution diffusion-weighted MRI. Magn. Reson. Med., 45(6):935-939, 2001. 
4. S. Geman and D. Geman. Stochastic relaxation, Gibbs distributions, and the Bayesian restoration of images. IEEE Transaction on Pattern Analysis and Machine Intelligence, PAMI-6(6):721-741, November 1984.

5. M. Koch, D. G. Norris, and M. Hund-Georgiadis. An investigation of functional and anatomical connectivity using diffusion tensor imaging. In ISMRM-ESMRMB, Glasgow, page 1509, 2001.

6. D Le Bihan. Diffusion and Perfusion Magnetic Resonance Imaging, chapter A-2-IV, pages 50-57. Raven Press, Ltd., New-York, 1995.

7. D. Le Bihan, J.-F. Mangin, C. Poupon, C. A. Clark, S. Pappata, N. Molko, and H. Chabriat. Diffusion tensor imaging: concepts and applications. Journal of Magnetic Reonance Imaging, 13:534-546, 2001.

8. G. J. M. Parker, C. A. Wheeler-Kingssbott, and G. J. Barker. Distributed anatomical brain connectivity derived from diffusion tensor imaging. In XVIIth IPMI, LNCS-2082, SpringerVerlag, pages 372-380, 2001.

9. C. Poupon, J.-F. Mangin, C. A. Clark, V. Frouin, J. Régis, D. Le Bihan, and I. Bloch. Towards inference of human brain connectivity from MR diffusion tensor data. Medical Image Analysis, 5:1-15, 2001.

10. D. S. Tuch, M. R. Wiegell, T. G. Reese, J. W. Belliveau, and J. Van Wedeen. Measuring cortico-cortical connectivity matrices with diffusion spectrum imaging. In ISMRMESMRMB, Glasgow, page 502, 2001.

11. C.-F. Westin, S.E. Maier, B. Khidir, P. Everett, F. A. Jolesz, and R. Kikinis. Image processing for diffusion tensor magnetic resonance imaging. In MICCAI'99, Cambridge, UK, LNCS1679, Springer-Verlag, pages 441-452, 1999.

12. M. R. Wiegell, H. B. Larsson, and V. J. Wedeen. Fiber crossing in human brain depicted with diffusion tensor MR imaging. Radiology, 217(3):897-903, 2000. 